\title{
A dual role for microglia in promoting tissue inhibitor of metalloproteinase (TIMP) expression in glial cells in response to neuroinflammatory stimuli
}

\author{
Jennifer V Welser-Alves ${ }^{1}$, Stephen J Crocker $^{2}$ and Richard Milner ${ }^{1 *}$
}

\begin{abstract}
Background: By neutralizing the effect of the matrix metalloproteinases (MMPs), the tissue inhibitors of matrix metalloproteinases (TIMPs) play a critical role in maintaining tissue proteolysis in balance. As the major reactive glial cell types in the central nervous system (CNS), microglia and astrocytes play fundamental roles in mediating tissue breakdown and repair. As such, it is important to define the TIMP expression profile in these cells, as well as the mechanisms of regulation by neuroinflammatory stimuli.

Methods: Primary mixed glial cultures (MGC), pure microglia, and pure astrocytes were used in this study. To study astrocytes, we employed a recently described pure astrocyte culture system, which has the major advantage of totally lacking microglia. The three different types of culture were treated with lipopolysaccharide (LPS) or individual cytokines, and cell culture supernatants assayed for TIMP-1 or TIMP-2 protein expression by western blot.

Results: LPS induced TIMP-1 expression in MGC, but not in pure astrocyte or microglial cultures. When pure astrocytes were treated with the cytokines IL-1 $1 \beta$, IFN- $\gamma$, TNF or TGF- $\beta 1$, only IL-1 $\beta$ induced TIMP-1 expression. Significantly, astrocyte TIMP-1 expression was restored in LPS-treated astrocyte cultures after the addition of microglia, or conditioned medium taken from LPS-activated microglia (MG-CM). Furthermore, this effect was lost after depletion of IL-1 $\beta$ from MG-CM. By contrast, TIMP-2 was constitutively expressed by astrocytes, whereas microglia expressed TIMP-2 only after exposure to serum.

Conclusions: Taken together, these results demonstrate an important concept in glial interactions, by showing that microglia play a central role in regulating glial cell expression of TIMPs, and identify microglial IL-1 $\beta$ as playing a key role in mediating microglial-astrocyte communication.
\end{abstract}

\section{Background}

By inhibiting the matrix metalloproteinases (MMPs), the tissue inhibitors of metalloproteinases (TIMPs) play an important role in maintaining proteolysis in balance [1-3]. In addition to this role, increasing evidence suggests that TIMPs also function in a MMP-independent manner, to regulate different aspects of cell behavior, including cell survival, cell proliferation, and the regulation of angiogenesis (reviewed in [4]).

\footnotetext{
* Correspondence: rmilner@scripps.edu

'Department of Molecular and Experimental Medicine, The Scripps Research Institute, 10550 North Torrey Pines Road, La Jolla, CA 92037, USA

Full list of author information is available at the end of the article
}

Within the central nervous system (CNS), MMPs and TIMPs play important roles, both during development, and during the pathogenesis and remodeling that follows CNS damage [5-8]. The two major reactive glial cell types in the CNS are microglia and astrocytes. In light of the major role these cells play during CNS disease and repair, it is a high priority to define which MMPs and TIMPs are expressed by these two glial cell types, and understand how this expression is regulated during disease pathogenesis. To shed some light on this, we previously examined TIMP expression at the mRNA level by RNase protection assay in primary glial cultures. This showed that while TIMP-2 is constitutively 
expressed, both in astrocyte-enriched cultures and microglia, TIMP-1 is much more restricted in its expression, being expressed in astrocyte-enriched cultures only after stimulation by IL- $1 \beta$ or lipopolysaccharide (LPS) [9]. In these previous studies, we took the currently acceptable standard approach of using mixed glial cultures (MGC) to represent astrocytes. Importantly though, as MGC contain microglia in addition to astrocytes, it was not possible to determine whether LPS and IL- $1 \beta$ exert a direct effect on astrocyte TIMP-1 production, or whether this effect requires the production of microglial intermediate factors. Indeed, in light of the finding that astrocytes express only marginal levels of the LPS receptor, TLR-4 [10], it seemed more likely that the influence of LPS was mediated via microglial factors. Based on these observations, we proposed the hypothesis that LPS promotes astrocyte TIMP-1 expression through an indirect mechanism, in which LPS induces microglia to secrete a soluble factor (possibly IL-1 $\beta$ ), which then stimulates astrocyte TIMP-1 expression. These previous findings indicate the existence of an important interplay between microglia and astrocytes in their regulation of gene expression. As it is becoming clear that astrocytes play important roles in regulating many aspects of CNS function, including myelination [11] and microglial reactivity [12], identifying how other CNS cell types influence astrocyte functions becomes an important priority.

To gain an improved understanding of the significance of microglial-astrocyte interactions in these responses, we recently developed and characterized a novel method of obtaining pure astrocytes, in which neurospheres containing neural stem cells (NSC) are differentiated into astrocytes in the presence of $10 \%$ fetal bovine serum (FBS) [13]. This approach yields pure astrocyte cultures, totally devoid of microglia, thus permitting an analysis of astrocyte protein expression, not complicated by the presence of contaminating microglia. We have already used this system to good effect to demonstrate that astrocytes and microglia show distinct, non-overlapping expression profiles of MMPs. Using this improved method, we found that in contrast to MGC, pure astrocyte cultures express a limited repertoire of MMPs, quite distinct from the microglial pattern, highlighted by the finding that microglia, not astrocytes express MMP9 [13]. This study also showed that in contrast to MGC or microglia, pure astrocytes appear to be unresponsive to LPS, showing no alterations in MMP expression patterns, consistent with the finding that astrocytes express only low levels of the LPS receptor, TLR-4 [10].

The purpose of the current study was to employ the improved pure astrocyte culture system, and together with pure microglial cultures, define the expression of TIMP-1 and TIMP-2 in astrocytes or microglia at the protein level, and examine how this is regulated by cytokines and LPS. Then, test our hypothesis that the effect of LPS on astrocyte TIMP-1 expression is mediated via a microglial-secreted factor, and if so, identify the molecular mechanism responsible.

\section{Methods}

\section{Animals}

The studies described have been reviewed and approved by The Scripps Research Institute Institutional Animal Care and Use Committee. All cell cultures were obtained from $\mathrm{C} 57 \mathrm{Bl} / 6$ mice which were maintained under pathogen-free conditions in the closed breeding colony of The Scripps Research Institute (TSRI).

\section{Cell culture}

Mixed glial cultures (MGC) were prepared from 0-2 day old C57Bl/6 mouse pups, as previously described [14], and maintained in poly-D-lysine coated T75 flasks in DMEM containing 10\% fetal bovine serum (FBS) (all from Sigma, St. Louis, MO). After 7-10 days, MGC were shaken for 30 minutes and the supernatant containing detached microglia was collected. Microglia were counted by hemocytometer, re-suspended in the shakeoff medium, and plated at a density of $2 \times 10^{5}$ cells/well in poly-D-lysine coated 6-well plates (Nunc, Naperville, IL). Microglial purity was $>99 \%$ as determined by Mac1 in flow cytometry. After MGC were harvested of microglial cells, the MGC were passaged into poly-Dlysine coated 6-well plates and maintained in DMEM containing 10\% FBS. Pure astrocyte cultures were prepared as previously described [13], by plating neurospheres into poly-D-lysine coated 6-well plates and maintained in DMEM containing 10\% FBS. Astrocyte purity of these neurosphere-derived cultures was > 99\% as determined by GFAP immunocytochemistry.

\section{Treatment of glial cultures with LPS and cytokines}

After microglia were shaken off MGC, they were allowed to attach overnight, before the medium was changed next day to serum-free DMEM containing N1supplement, L-glutamine and penicillin/streptomycin (all from Sigma). In experiments designed to evaluate the role of microglia in promoting TIMP-1 expression in astrocytes, $0.5 \times 10^{5}$ microglia were added per well of astrocytes. MGC and pure astrocyte cultures were maintained in DMEM containing 10\% FBS until confluency, then switched to the same N1-supplemented serum-free medium. Upon switching to serum-free medium, all three types of culture received either no treatment, lipopolysaccharide (LPS, serotype: E. Coli 0111.B4, $1 \mu \mathrm{g} / \mathrm{ml}$, Sigma), IFN- $\gamma(10 \mathrm{U} / \mathrm{ml}, \mathrm{R} \& \mathrm{D}$, Minneapolis, MN), IL-1 $\beta$ (10 ng/ml, Peprotech, Rocky Hill, NJ), TNF- $\alpha$ (20 ng/ ml, Genentech, San Francisco, CA), or TGF- $\beta 1$ (20 ng/ 
ml, R\&D). After 2 days treatment, cell culture supernatants were collected for analysis. LPS-activated microglial conditioned medium (MG-CM) was produced and collected the same way, and filtered through a $0.22 \mu \mathrm{m}$ filter before addition to pure astrocyte cultures.

\section{Antibodies}

The goat polyclonal antibodies specific for TIMP-1 (AF980), TIMP-2 (AF-971), and IL-1 $\beta$ (AF-401-NA) were obtained from R\&D Systems. Sources of other antibodies include: rabbit anti-goat polyclonal antibody (Vector Labs, Burlingame, CA), mouse anti-GFAP-Cy3 (Sigma, cat. no. C9205, clone G-A-5), rat anti-Mac-1 antibody (BD Pharmingen, La Jolla, CA, cat. no. 553310, clone M1/70), anti-rat-Alexafluor 488 (Invitrogen, Carlsbad, CA), and anti-goat-HRP (Pierce, Rockford, IL).

\section{Immunocytochemistry}

To investigate the purity of the different glial culture systems, cells were cultured on fibronectin coated glass coverslips in DMEM containing 10\% FBS. Cells were washed in PBS and fixed in acid/alcohol (95:5) at $-20^{\circ} \mathrm{C}$ for 10 minutes, before being washed extensively and blocked in 5\% normal goat serum (NGS) in PBS for 30 minutes. Cells were then incubated with a Mac-1 monoclonal antibody for 1 hour at room temperature, washed and then incubated with anti-rat-Alexafluor 488 for 30 minutes at room temperature. After washing, cells were incubated with a mouse anti-GFAP-Cy3 conjugated antibody for 1 hour, washed and then incubated with the nuclear Hoechst stain (Sigma) for 10 minutes. Coverslips were then washed and mounted in aquamount (Polysciences, Warrington, PA).

\section{Immunodepletion experiments}

LPS-activated MG-CM was immunodepleted of IL-1 $\beta$ by six successive rounds of immunoprecipitation. Briefly, the cell culture supernatants were first pre-cleared for one hour with $30 \mu \mathrm{l}$ of protein A sepharose beads per $\mathrm{ml}$ of cell lysate. This was followed by six consecutive immunoprecipitations, in which $4 \mu$ of goat anti-IL-1 $\beta$ and $4 \mu \mathrm{l}$ of rabbit anti-goat antibody were added to a tube containing $1 \mathrm{ml} \mathrm{MG-CM}$ and $30 \mu \mathrm{l}$ beads. Each ID was performed for a minimum period of 4 hours, all at $4^{\circ} \mathrm{C}$ on a rotating platform. For control, the same MG$\mathrm{CM}$ also underwent a mock immunodepletion containing $4 \mu \mathrm{l}$ of normal goat serum and $4 \mu \mathrm{l}$ of rabbit antigoat antibody. After the last ID, the supernatants were sterile filtered through a $0.22 \mu \mathrm{m}$ filter before addition to pure astrocyte cultures.

\section{Western blotting}

Cell culture supernatants were boiled in reducing sample buffer for 5 minutes before being analysed by $12 \%$
SDS-PAGE (Invitrogen) under reduced conditions. $10 \mu \mathrm{l}$ of cell culture supernatants were electro-blotted for 1 hour onto nitrocellulose membranes (Invitrogen), blocked overnight in 3\% non-fat milk in TBS containing $0.1 \%$ Tween-20 (Sigma) and probed with the anti-TIMP or anti-IL-1 $\beta$ antibodies for one hour, before being washed, then probed with anti-goat-HRP conjugate (Pierce) for one hour, and then washed extensively. Protein bands were visualised with the SuperSignal WestFemto ECL detection system (Pierce) according to the manufacturers' instructions. Band intensity was quantified using the BioRad Imaging system. Results represent the mean \pm SEM of 4 experiments, with each sample examined in triplicate within each experiment. Statistical significance was assessed by using Student's t test, in which $\mathrm{p}<0.05$ was defined as statistically significant.

\section{Results}

LPS induces TIMP-1 expression in mixed glial cultures, but not pure cultures of astrocytes or microglia

In a previous study, we showed that LPS and IL- $1 \beta$ promote TIMP-1 expression in mixed glial cultures (MGC), containing astrocytes and microglia, but not in pure microglial cultures [9]. From this we hypothesized that LPS promotes microglia to express soluble factors (possibly IL-1 $\beta$ and/or other factors), which then stimulate TIMP-1 expression in astrocytes. As the MGC system contains microglia in addition to astrocytes, it was not possible to directly test this idea using the MGC system. However, with the advent of our recently described pure astrocyte culture system, which is entirely free of microglia [13], it now becomes possible to perform a molecular dissection of the signals mediated between microglia and astrocytes. The three different glial culture systems are shown in Figure 1, with astrocytes and microglia identified by GFAP and Mac-1 positivity, respectively. MGC contained both astrocytes and microglia, pure astrocyte cultures were entirely devoid of microglia, while pure microglia cultures contained no astrocytes. All experiments were performed under serum-free conditions, and TIMP protein expression examined by western blot of cell culture supernatants. In the first experiment, we examined TIMP-1 expression in three different types of culture: MGC, pure astrocytes, or pure microglia, with or without the addition of LPS. After 2 days incubation, TIMP-1 expression was examined. As shown in Figure 2A, TIMP-1 expression was never seen in any of the three types of culture untreated with LPS, and of the LPS-treated cells, only the MGC expressed TIMP-1 protein (running at the predicted molecular weight of $28 \mathrm{kD}$ ). This result confirmed our previous observation (performed at the mRNA level), showing that MGC express TIMP-1 in response to LPS stimulation, but microglia do not, and also make the important 


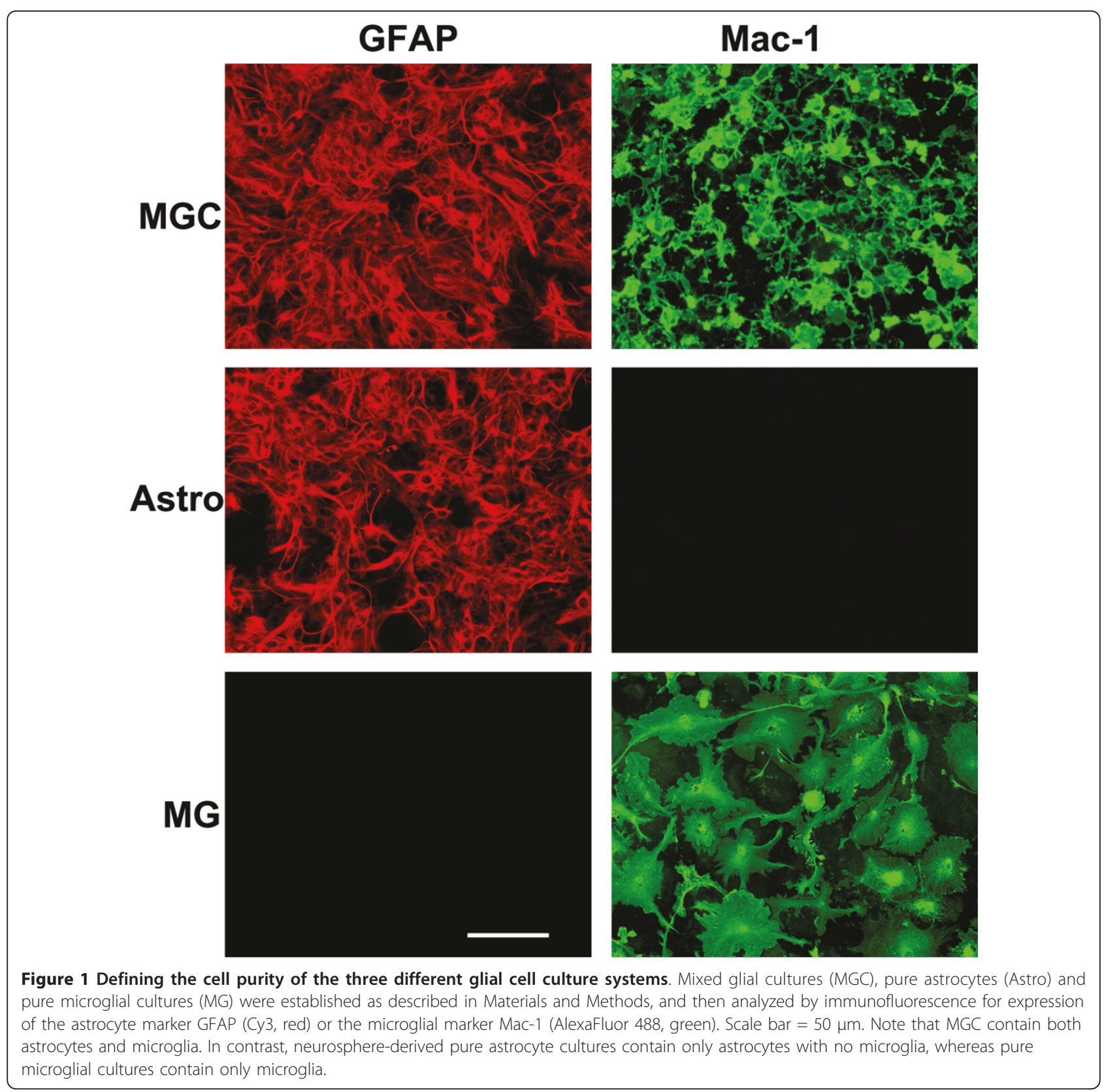

point that pure astrocyte cultures are unresponsive to LPS stimulation. As MGC contain a mix of astrocytes and microglia, these results suggest that microglia are required to mediate the influence of LPS on TIMP-1 expression in astrocytes.

Pure astrocytes express TIMP-1 in the presence of LPSactivated microglia conditioned medium

To directly test whether microglia or their secreted factors influence astrocyte TIMP-1 expression, we cultured pure astrocytes either alone, in the presence of added microglial cells, or in the presence of microglia conditioned medium (MG-CM) previously stimulated by LPS, all cultures being stimulated with LPS. As shown in Figure 2B, whereas pure astrocyte cultures treated with LPS expressed no TIMP-1, the addition of microglial cells or LPS-activated MG-CM promoted robust TIMP-1 expression. This result confirms that microglia are required to mediate the effect of LPS on astrocyte TIMP1 expression, and furthermore shows that the effect is mediated via a microglial soluble factor. Based on our previous finding that of a panel of different cytokines tested, only IL-1 $\beta$ elevated TIMP-1 mRNA levels in MGC [9], we investigated a number of different cytokines 

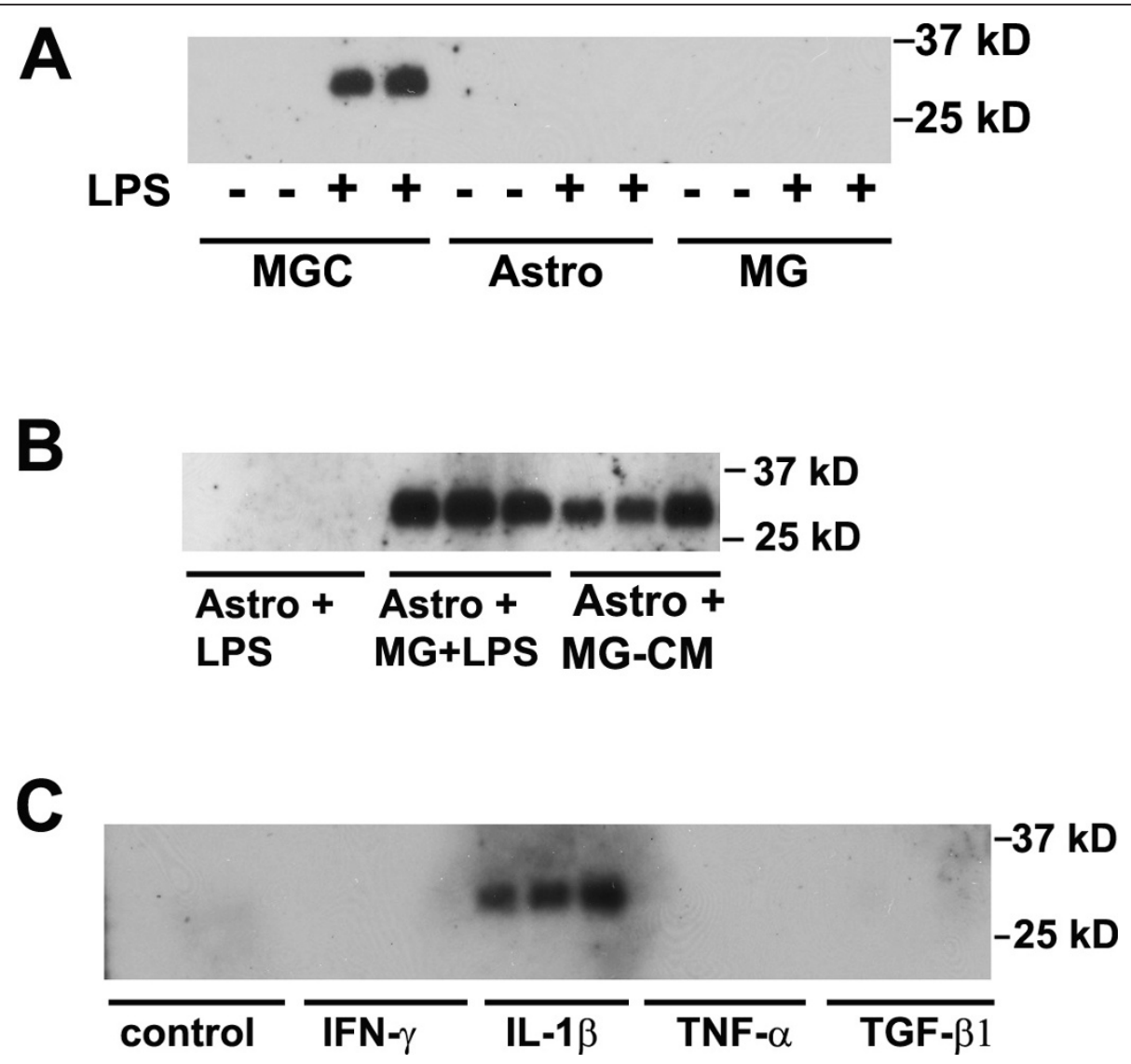

Figure 2 Analysis of glial cell TIMP-1 expression in response to cytokines and LPS. TIMP-1 expression was examined by western blot of cell culture supernatants, as described in Materials and Methods. A. The influence of LPS on TIMP-1 expression in the three different types of glial culture. MGC, astrocytes, and microglial cultures were treated with LPS for 2 days, then cell culture supernatants examined for TIMP-1 expression. Note that cultures not treated with LPS did not express TIMP-1, and TIMP-1 protein ( 28 kD band) was induced only in MGC following LPS stimulation. B. TIMP-1 induction in astrocytes in the presence of activated microglia or MG-CM. Pure astrocyte cultures received either: LPS, microglia + LPS, or LPS-activated MG-CM. Note that the presence of LPS-activated microglia or LPS-activated MG-CM strongly induced TIMP-1 expression in astrocytes. C. The influence of cytokines on TIMP-1 expression by astrocytes. Pure astrocyte cultures were treated with a panel of different cytokines for 2 days, and the supernatants analyzed for TIMP-1 expression. Note that of the cytokines tested, only IL-1 $\beta$ stimulated TIMP-1 expression in astrocytes.

for their effect to stimulate TIMP-1 expression in pure astrocyte cultures. Pure astrocytes were treated with IL$1 \beta$, IFN- $\gamma$, TNF or TGF- $\beta 1$, their supernatants collected after 2 days, and examined for TIMP-1 expression (see Figure 2C). Consistent with our previous mRNA data, this demonstrated that IL-1 $\beta$ was the only factor that promoted TIMP-1 in pure astrocytes, and furthermore, showed that none of the cytokines stimulated TIMP-1 expression in microglial cultures (not shown).

$\mathrm{IL}-1 \beta$ is the dominant microglial factor responsible for mediating astrocyte TIMP-1 expression in response to LPS Having confirmed that IL- $1 \beta$ has a direct effect on TIMP-1 induction in astrocytes, we next addressed the question: is IL-1 $\beta$ the major microglial factor that mediates the LPS-induction of astrocyte TIMP-1 expression? To answer this question, we took LPS-activated MG-CM and removed all the available IL- $1 \beta$ by performing six successive rounds of immunodepletion with a polyclonal anti-IL-1 $\beta$ antibody. Following this procedure, we first established that the immunodepletion had worked, by examining IL-1 $\beta$ levels in the MG-CM, before and after this procedure. As shown in Figure $3 \mathrm{~A}$, IL-1 $\beta$ was detected in the MG-CM prior to, but not after IL-1 $\beta$ immunodepletion, demonstrating that this procedure effectively removed all IL- $1 \beta$ from the MG-CM. Next, we examined TIMP-1 expression in pure astrocyte cultures treated with LPS-activated MG-CM, in which the MG$\mathrm{CM}$ had either been mock-depleted (control) or really depleted of IL-1 $\beta$. As shown in Figures $3 B$ and $3 C$, this demonstrated that removal of IL- $1 \beta$ from the LPS-activated MG-CM significantly reduced astrocyte TIMP-1 expression (from $365.6 \pm 31.0$ under control conditions to $91.2 \pm 36.3$ units after IL- $1 \beta$ immunodepletion, $\mathrm{p}<$ 


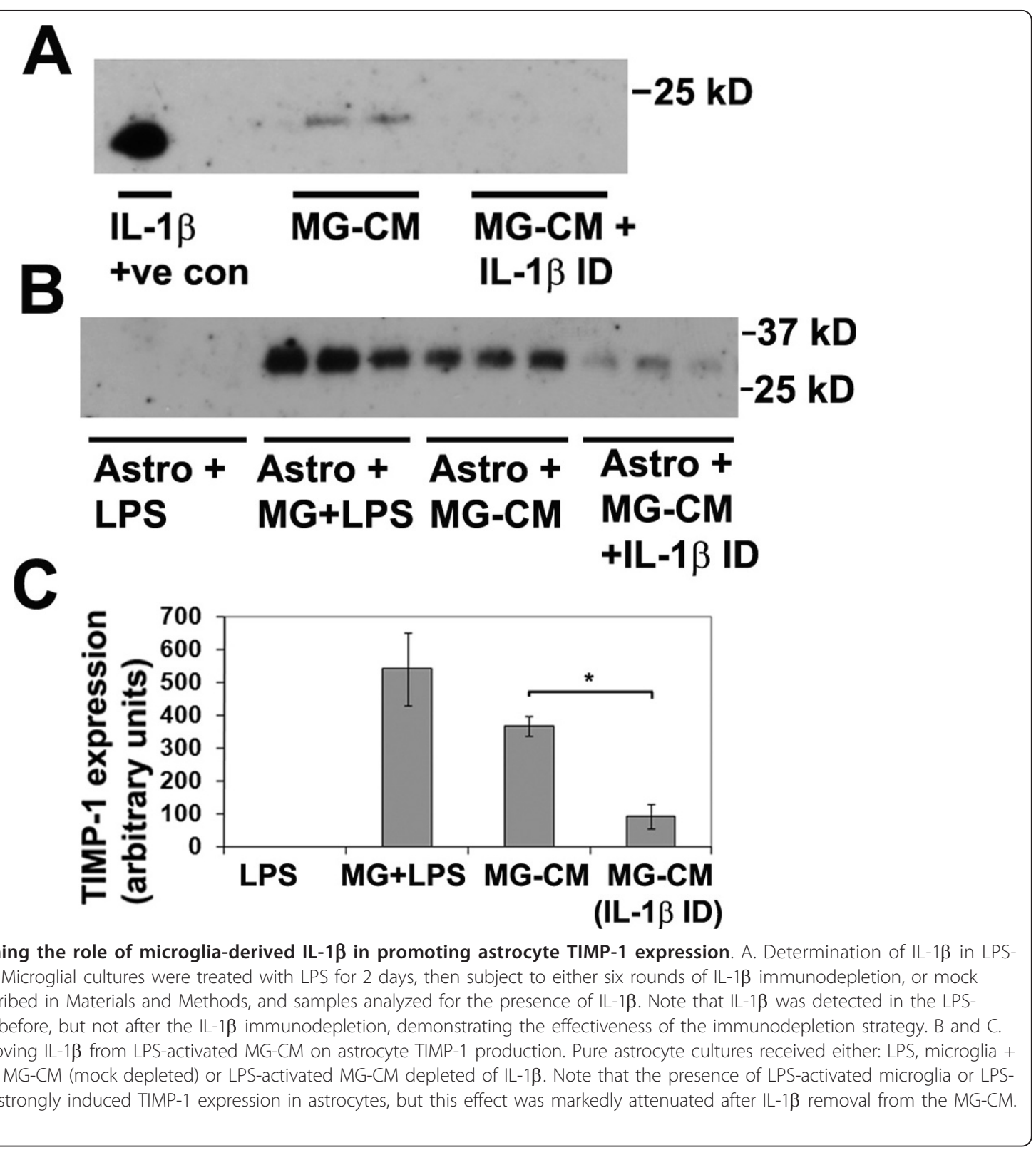

0.02 ) demonstrating that IL- $1 \beta$ is the dominant microglial factor responsible for mediating astrocyte TIMP-1 expression in response to LPS

Under serum-free conditions, astrocytes but not microglia constitutively express TIMP-2

In parallel with our analysis of TIMP-1 expression in astrocyte and microglial cultures, we also examined the expression of TIMP-2 in these cultures. In contrast to TIMP-1 expression, this demonstrated that TIMP-2 was expressed at high levels both by MGC and pure astrocytes, but not microglia, and furthermore, not affected by LPS (see Figure 4A). This confirms that astrocytes constitutively express TIMP-2. To examine whether
TIMP-2 expression was regulated by cytokines, we examined the same panel of cytokines, as was used in the TIMP-1 experiment. This showed that TIMP-2 expression in MGC or in pure astrocyte cultures was not affected by any of the cytokines tested (see Figure $4 \mathrm{~B})$. Furthermore, none of the cytokines tested induced TIMP-2 expression in microglia (not shown).

Serum induces TIMP-2 expression in microglial cultures Our current study failed to show any TIMP-2 expression in microglia. This was unexpected in light of our previous findings using an RNase protection assay approach, which demonstrated TIMP-2 expression, both in MGC and in microglia. In fact, this data showed that 


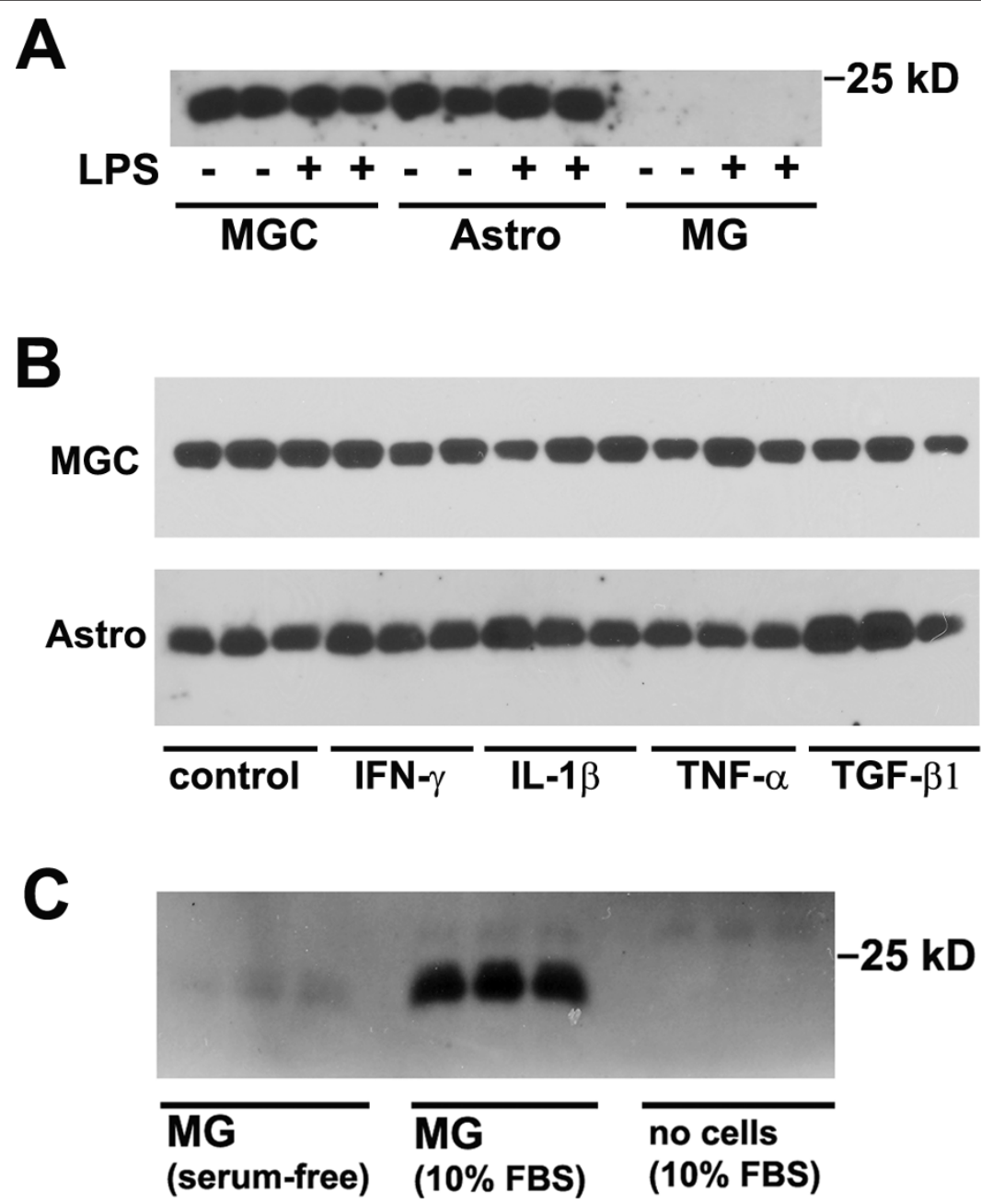

Figure 4 Analysis of glial cell TIMP-2 expression in response to cytokines and LPS. TIMP-2 expression was examined by western blot of cell culture supernatants, as described in Materials and Methods. A. The influence of LPS on TIMP-1 expression in the three different types of glial culture. MGC, astrocytes, and microglial cultures were treated with LPS for 2 days, then cell culture supernatants examined for TIMP-2 expression. Note that MGC and pure astrocytes constitutively expressed TIMP-2 ( 22 kD) band, and this expression was not enhanced by LPS stimulation. In contrast, microglial cultures did not express TIMP-2. B. The influence of cytokines on TIMP-2 expression by MGC and pure astrocytes. MGC and pure astrocyte cultures were treated with a panel of different cytokines for 2 days, and the supernatants analyzed for TIMP1 expression. Note that both MGC and pure astrocytes constitutively expressed TIMP-2, and this expression level was not significantly affected by any of the cytokines tested. C. The influence of serum on microglial TIMP-2 expression. Pure microglial cultures were grown either in serum-free medium or in medium containing 10\% FBS. After 2 days, the cell culture supernatants were analyzed for TIMP-2 expression. Note that the presence of serum strongly induced TIMP-2 expression in microglial cells.

TIMP-2 was the only TIMP expressed by microglia [9]. In trying to pinpoint the reason for this discrepancy, we noted that in the first study, the cell cultures were maintained in serum-containing medium (10\% FBS), while in the current study, cells were maintained under serumfree conditions. To test whether serum accounted for the difference between the two studies in microglial TIMP-2 expression, we cultured microglia in either serum-free or serum-containing media for two days, then examined TIMP-2 expression by western blot. As shown in Figure $4 \mathrm{C}$, in the absence of serum, microglia expressed no TIMP-2 protein, while in the presence of serum, a dramatic increase in TIMP-2 expression was observed. To exclude the possibility that the TIMP-2 protein was serum-derived, we also examined the presence of TIMP-2 in the 10\% FBS-containing medium that the cells were grown in; this failed to show any TIMP-2 protein. Therefore we conclude that serum strongly induces TIMP-2 expression in microglial cells.

\section{Discussion}

In this study we used pure cultures of astrocytes or microglia to examine the expression of TIMP-1 and TIMP-2 at the protein level. Consistent with our 
previous study, which examined these events at the mRNA level [9], we found that LPS induced TIMP-1 expression in astrocyte-enriched MGC (which contain microglia in addition to astrocytes), but interestingly, this effect was not seen in pure astrocyte or microglial cultures. Significantly, astrocyte TIMP-1 expression was restored in pure astrocyte cultures after the addition of microglia, or conditioned medium taken from LPS-activated microglia (MG-CM), and this effect was lost after depletion of IL-1 $\beta$ from MG-CM. In contrast, TIMP-2 was constitutively expressed by astrocytes, while microglia expressed TIMP-2 only after exposure to serum. Taken together, these results demonstrate an important concept in glial interactions, by showing that microglia play a central role in regulating glial cell expression of TIMPs. Most importantly, they provide direct evidence that LPS promotes astrocyte TIMP-1 expression via an indirect mechanism in which LPS stimulates microglia to secrete IL-1 $\beta$, which then induces astrocyte TIMP-1 expression.

\section{Functions of TIMPs in the CNS}

The TIMP family is comprised of four members, TIMP1 through TIMP-4 $[1,2,4]$. TIMPs were initially characterized as naturally-occurring inhibitors of the MMPs that were important for the maintenance of proteolytic balance and as such play important roles in a number of developmental and physiological remodelling processes, including cell migration, wound healing, neuronal survival and angiogenesis (reviewed in $[1,2,4])$. The importance of the protective MMP-neutralizing effect of TIMP-1 in the CNS is best illustrated by the finding that TIMP-1 knockout mice display increased levels of MMP-9 activity, BBB breakdown and size of ischemic insult following focal cerebral ischemia [15]. In support of this, a parallel study revealed that adenoviral delivery of TIMP-1 or TIMP-2 resulted in reduced levels of neuronal death in a model of global cerebral ischemia [16]. TIMP-1 also plays an important MMP-dependent role in demyelinating disease, as illustrated by increased levels of demyelination and myelin pathology in TIMP-1 deficient mice in the mouse model of multiple sclerosis, experimental autoimmune encephalomyelitis (EAE) [17]. In addition to the well-described MMP-dependent actions, in more recent years, several studies have demonstrated that TIMPs also have a number of MMPindependent actions, that include the regulation of cell growth and cell death (reviewed in [4]). Specifically, it has been shown that TIMP-2 promotes neuronal differentiation by inhibiting cell proliferation, in an MMPindependent manner $[18,19]$. Exactly how these MMPindependent effects are mediated at the molecular level is still to be determined. However, studies on endothelial cells, highly relevant because of the influence of
TIMPs on angiogenesis, suggest that TIMPs can bind to a variety of cell surface receptors, including the vascular endothelial growth factor (VEGF) receptor-2 [20], and the $\alpha 3 \beta 1$ integrin [21], raising the possibility that TIMPs signal through these specific receptors.

Recent studies have described two unexpected roles for TIMP-1 in the regulation of neural cell behaviour. First, TIMP-1 influences the growth and morphology of cortical neurons in an MMP-dependent manner; recombinant TIMP-1 reduced neurite length while dramatically increasing the size of growth cones [22]. Second, a series of studies have demonstrated that TIMP-1 plays an important role in the generation and differentiation of oligodendrocytes [23]. TIMP-1 knockout mice show reduced levels of myelin after induction of EAE [17], whereas mice that express higher levels of TIMP-1 in their CNS exhibit attenuated demyelination in EAE [24]. This is supported by the observations that TIMP-1 KO mice show delayed oligodendrocyte differentiation during development [23], and that TIMP-1 KO neural stem cells (NSC) yield significantly fewer oligodendrocytes compared to wild type, an effect that can be reversed by the addition of recombinant TIMP-1 [23].

\section{TIMP expression by astrocytes and microglia}

In vivo studies show that TIMP expression is more strongly associated with astrocytes than microglia $[25,26]$. In particular, the consensus view is that TIMP-1 expression is astrocyte-specific, and this expression is highly upregulated during neuroinflammatory states involving breakdown of the blood-brain barrier and leukocyte infiltration into the CNS [27-30], suggesting that astrocyte TIMP-1 may play an important role in curtailing the influx of activated infiltrating leukocytes, by inhibiting the action of the proteolytic enzyme, MMP-9. This idea is supported by the finding that in a mouse model of focal cerebral ischemia, TIMP-1 knockout mice display increased levels of MMP-9 activity, BBB breakdown and size of ischemic insult [15]. In addition to TIMP-1, astrocyte expression of TIMP-2 has also been described in vivo following astrocyte activation after brain injury [31,32]. Interestingly, TIMP-2 expression has also been described in microglia following brain trauma [33]. These findings are in keeping with our own observations, namely that TIMP-1 appears to be astrocyte-specific, but TIMP-2 can be expressed by both astrocytes and microglia.

A small number of studies have also examined TIMP expression in pure microglial cultures, with mixed results. One study performed with PCR on human primary microglia described all four TIMP family members within microglia [34], and an ELISA study using the human microglial cell line CHME3 described both TIMP-1 and TIMP-2 present in microglia [35]. In 
contrast, examination of primary mouse glial cultures showed that TIMP-1 protein was only present in astrocytes, not microglia [36], and our prior study using RNAse protection assay was entirely consistent with this finding [9]. Indeed, our current finding lends further weight to this idea by showing that microglia expressed no TIMPs under serum-free conditions, but show strong induction of TIMP-2 only after exposure to serum. This finding has two important implications. First, it suggests that some of the differences apparent between the studies of microglial TIMP expression may be explained by the use of serum in microglial cultures. All of the above described studies (including our RNAse protection assay analysis) used serum-containing media, which may not be the best representation of the in vivo condition. Of course, it is also possible that species-species variation may contribute to these differences. Second, as our data clearly demonstrates that serum strongly promotes TIMP-2 expression in microglia, this implies that breakdown of the BBB will trigger microglial TIMP-2 expression, thereby limiting the destructive power of the oncoming wave of MMP-9 introduced into the breached CNS. This is remarkably similar to our previously described serum-induction of TIMP-1 expression in astrocytes [9]. Taken together, it suggests that introduction of serum into the brain induces TIMP expression in both astrocytes (TIMP-1) and microglia (TIMP-2) as part of an intrinsic damage limitation program.

One clear point to emerge from this study is that the bacterial toxin, LPS does not directly stimulate TIMP-1 expression in astrocytes; rather it mediates its effect by inducing microglia IL- $1 \beta$ expression, which then triggers astrocyte TIMP-1 expression. This is important because it demonstrates that microglia play a pivotal protective role in driving glial TIMP expression in two ways, first, by upregulating TIMP-2 in response to serum, and second, by facilitating astrocyte TIMP-1 expression is response to bacterial infection. It also shows that the pro-inflammatory cytokine IL- $1 \beta$ has two sides to its character; having not only deleterious effects in the CNS, but also providing certain important protective functions. In the current study we have shown that IL$1 \beta$ is the major microglial secreted factor responsible for triggering astrocyte TIMP-1 expression. Removal of IL$1 \beta$ resulted in a $75 \%$ reduction in astrocyte TIMP-1 expression. That astrocytes still showed a small TIMP-1 response might be due to the presence of another factor within the MG-CM, or alternatively, it could be due to a failure of our immunodepletion strategy to totally remove all IL-1 $\beta$ from the MG-CM. In fact, the latter seems more likely, as it is almost impossible to totally remove a factor, so that even a small amount of IL-1 $\beta$ might be capable of eliciting some degree of TIMP-1 response. If true, this suggests that neuroinflammation in IL-1 $\beta$ knockout mice may result in an absent astrocyte TIMP-1 response, and a potentially much stronger MMP-proteolytic effect.

\section{Conclusions}

The aim of this study was to examine whether TIMP-1 and TIMP-2 are expressed in pure cultures of astrocytes and microglia, and then define the influence of LPS and cytokines on this expression. While LPS induced TIMP-1 expression in astrocyte-enriched MGC (which contain microglia in addition to astrocytes), this effect was not seen in pure astrocyte or microglial cultures. However, the addition of microglia, or conditioned medium taken from LPS-activated microglia (MG-CM) restored astrocyte TIMP-1 expression, and this effect was lost after depletion of IL-1 $\beta$ from MG-CM. By contrast, TIMP-2 was constitutively expressed by astrocytes, and not altered by any of the factors tested, while microglia expressed TIMP-2 only after exposure to serum. These results demonstrate an important concept in glial interactions, by showing that microglia play a central role in regulating glial cell expression of TIMPs, and identify microglial IL-1 $\beta$ as playing a key role in mediating microglial-astrocyte communication.

\section{Acknowledgements}

This work was supported by the National Multiple Sclerosis Society: by a Harry Weaver Neuroscience Scholar Award to RM (JF 2125A1/1), a Career Transition Award to SJC (TA 3021 A1/1T), and a Post-Doctoral Fellowship to JWW (FG 1879-A-1), and by NIH grant RO1 NS060770. This is manuscript number 21213 from The Scripps Research Institute.

\section{Author details}

1 Department of Molecular and Experimental Medicine, The Scripps Research Institute, 10550 North Torrey Pines Road, La Jolla, CA 92037, USA.

${ }^{2}$ Department of Neuroscience, University of Connecticut Health Center, Farmington, $C T$, USA.

\section{Authors' contributions}

JW carried out the biochemical analysis and contributed to drafting the manuscript. SJC participated in the design of the study and also assisted in manuscript preparation. RM conceived of the study, prepared the cell cultures, and drafted the manuscript. All authors read and approved the final manuscript.

\section{Competing interests}

The authors declare that they have no competing interests.

Received: 6 April 2011 Accepted: 1 June 2011 Published: 1 June 2011

\section{References}

1. Crocker SJ, Pagenstecher A, Campbell IL: The TIMPs tango with MMPs and more in the central nervous system. J Neurosci Res 2004, 75:1-11.

2. Gardner J, Ghorpade A: Tissue inhibitor of metalloproteinase (TIMP)-1: the TIMPed balance of matrix metalloproteinases in the central nervous system. J Neurosci Res 2003, 74:801-806

3. Agrawal SM, Lau L, Yong WW: MMPs in the central nervous system: where the good guys go bad. Semin Cell Dev Biol 2008, 19:42-51.

4. Stetler-Stevenson WG: Tissue inhibitors of metalloproteinases in cell signaling: metalloproteinases-independent biological activities. Sci Signal 2008, 1:re6. 
5. Candelario-Jalil E, Yang Y, Rosenberg GA: Diverse roles of matrixmetalloproteinsases and tissue inhibitors of metalloproteinases in neuroinflammation and cerebral ischemia. Neuroscience 2009 158:983-994

6. Cunningham LA, Wetzel M, Rosenberg GA: Multiple roles for MMPs and TIMPs in cerebral ischemia. Glia 2005, 50:329-339.

7. Rosell A, Lo EH: Multiphasic roles for matrix metalloproteinases after stroke. Curr Opin Pharmacol 2008, 8:82-89.

8. Zhao BQ, Wang S, Kim hY, Storrie H, Rosen BR, Mooney DJ, Wang XL, Lo EH: Role of matrix metalloproteinases in delayed cortical responses after stroke. Nat Med 2006, 12:441-445.

9. Crocker SJ, Milner R, Pham-Mitchell N, Campbell IL: Cell and agonistspecific regulation of genes for matrix metalloproteinases and their tissue inhibitors by primary glial cells. J Neurochem 2006, 98:812-823.

10. Jack CS, Arbour N, Manusow J, Montgrain V, Blain M, McCrea E, Shapiro A, Antel JP: TLR signaling tailors innate immune responses in human microglia and astrocytes. J Immunol 2005, 175:4320-4330.

11. Watkins TA, Emery B, Mulinyawe S, Barres BA: Distinct stages of myelination regulated by gamma-secretase and astrocytes in a rapidly myelinating CNS coculture system. Neuron 2008, 60:555-569.

12. Lee M, Schwab C, McGeer PL: Astocytes are GAGAergic cells that modulate microglial activity. Glia 2011, 59:152-165.

13. Crocker SJ, Frausto RF, Whitton JL, Milner R: A novel method to establish microglia-free astrocyte cultures: comparison of matrix metalloproteinase expression profiles in pure cultures of astrocytes and microglia. Glia 2008, 56:1187-1198.

14. Milner R, Hung S, Wang X, Berg G, Spatz M, del Zoppo G: Responses of endothelial cell and astrocyte matrix-integrin receptors to ischemia mimic those observed in the neurovascular unit. Stroke 2008, 39:191-197.

15. Fujimoto M, Takagi Y, Aoki T, Harase M, Marumo T, Gomi M, Nishimura M, Kataoka H, Hashimoto N, Nozaki K: Tissue inhibitor of metalloproteinases protect blood-btain barrier disruption in focal cerebral ischemia. J Cereb Blood Flow Metab 2008, 28:1674-1685

16. Magnoni S, Baker A, Thomson S, Jordan G, George SJ, McColl BW, McCulloch J, Horsburgh K: Neuroprotective effect of adenoviral-mediated gene transfer of TIMP-1 and -2 in ischemic brain injury. Gene Ther 2007, 14:621-625.

17. Crocker SJ, Whitmire JK, Frausto RF, Chertboonmuang P, Soloway PD, Whitton $J$, Campbell IL: Persistent macrophage/microglial activation and myelin disruption after experimental autoimmune encephalomyelitis in tissue inhibitor of metalloproteinase-1-deficient mice. Am J Pathol 2006 169:2104-2116

18. Perez-Martinez $L$, Jaworski DM: Tissue inhibitor of metalloproteinase-2 promotes neuronal differentiation by acting as an anti-mitogenic signal. J Neurosci 2005, 25:4917-4929.

19. Jaworski DM, Perez-Martinez L: Tissue inhibitor of metalloproteinase-2 (TIMP-2) expression is regulated by multiple neural differentiation signals. J Neurochem 2006, 98:234-247.

20. Qi JH, Ebrahem Q, Moore N, Murphy G, Claesson-Welsh L, Bond M, Baker A, Anand-Apte B: A novel function for tissue inhibitor of metalloproteinases-3 (TIMP-3): inhbition of angiogenesis by blockage of VEGF binding to VEGF receptor-2. Nat Med 2003, 9:407-415.

21. Seo DW, Li H, Guedez L, Wingfield PT, Diaz T, Salloum R, Wei BY, StetlerStevenson WG: TIMP-2 mediated inhbition of angiogenesis: an MMPindependent mechanism. Cell 2003, 114:171-180.

22. Ould-yahoui A, Tremblay E, Sbai O, Ferhat L, Bernard A, Charrat E, Gueye $Y$, Lim NH, Brew K, Risso JJ, Dive V, Khrestchatisky M, Rivera S: A new role for TIMP-1 in modulating neurite outgrowth and morphology of cortical neurons. PLoS One 2009, 4:e8289.

23. Moore C, Milner R, Nishiyama A, Frausto F, Serwanski D, Pagarigan R, Whitton JL, Miller R, Crocker SJ: Astrocytic TIMP-1 promotes oligodendrocyte differentiation and enhances CNS myelination. $J$ Neurosci 2011, 31:6247-54.

24. Althoff GE, Wolfer DP, Timmesfeld N, Kanzler B, Schrewe H, Pagenstecher A: Long-term expression of tissue inhibitor of matrix metalloproteinase- 1 in the murine central nervous system does not alter the morphological and behavioral phenotype but alleviates the course of experimental allergic encephalomyelitis. Am J Pathol 2010, 177:840-853.

25. Rivera S, Ogier C, Jourquin J, Timsit S, Szklarcyzk AW, Miller KM, Gearing AJ, Kaczmarek L, Khrestchatisky M: Gelatinase B and TIMP-1 are regulated in a cell- and time-dependent manner in association with neuronal death and glial reactivity after global forebrain ischemia. Eur J Neurosci 2002, 15:19-32.

26. Bugno M, Witek B, Bereta J, Bereta M, Edwards DR, Kordula T: Reprogramming of TIMP-1 and TIMP-3 expression profiles in brain microvascular endothelial cells and astrocytes in response to proinflammatory cytokines. FEBS Lett 1999, 448:9-14.

27. Pagenstecher A, Lassmann S, Carson MJ, Kincaid CL, Stalder AK Campbell IL: Astrocyte-targeted expression of IL-12 induces active cellular immune responses in the central nervous system and modulates experimental allergic encephalomyelitis. J Immunol 2000, 164:4481-4492.

28. Pagenstecher A, Stalder AK, Kincaid CL, Shapiro L, Campbell IL: Differential expression of matrix metalloproteinase and tissue inhibitor of matrix metalloproteinase genes in the mouse central nervous system in normal and inflammatory states. Am J Pathol 1998, 152:729-741.

29. Teesalu $T$, Hinkkanen $A E$, Vaheri $A$ : Coordinated induction of extracellular proteolysis systems during experimental autoimmune encephalomyelitis in mice. Am J Pathol 2001, 159:2227-2237.

30. Clark RT, Philip-Nance J, Noor S, Wilson EH: T-cell production of matrix metalloproteinases and inhibition of parasite clearance by TIMP-1 during chronic Toxoplasma infection in the brain. ASN Neuro 2011, 3:pii: e00049.

31. Muir EM, Adcock KH, Morgenstern DA, Clayton R, von Stillfried N, Rhodes K, ellis C, Fawcett JW, Rogers JH: Matrix metallproteinases and their inhbitors are produced by overlapping populations of activated astrocytes. Brain Res Mol Brain Res 2002, 100:103-117.

32. Agapova OA, Ricard CS, Salvador-Silva M, Hernandez MR: Expression of matrix metalloproteinases and tissue inhibitors of metalloproteinases in human optic nerve head astrocytes. Glia 2001, 33:205-216.

33. Jaworski DM: Differential regulation of tissue inhibitor of metalloproteinase mRNA expression in response to intracranial injury Glia 2000, 30:199-208.

34. Nuttall RK, Silva C, Hader W, Bar-Or A, Patel KD, Edwards DR, Yong WW: metalloproteinases are enriched in microglia compared with leukocytes and they regulate cytokine levels in activated microglia. Glia 2007, 55:516-526.

35. Cross AK, Woodroofe MN: Chemokine modulation of matrix metalloproteinase and TIMP production in adult rat brain microglia and a human microglial cell line in vitro. Glia 1999, 28:183-189.

36. Kumnok J, Ulrich R, Wewetzer K, Rohn K, Hansmann F, Baumgartner W, Alldinger S: Differential transcription of matrix-metalloproteinase genes in primary mouse astrocytes and microglia infected with Theiler's murine encephalomyeleitis virus. J Neurovirol 2008, 14:205-217.

doi:10.1186/1742-2094-8-61

Cite this article as: Welser-Alves et al.: A dual role for microglia in promoting tissue inhibitor of metalloproteinase (TIMP) expression in glial cells in response to neuroinflammatory stimuli. Journal of Neuroinflammation 2011 8:61.

\section{Submit your next manuscript to BioMed Central and take full advantage of:}

- Convenient online submission

- Thorough peer review

- No space constraints or color figure charges

- Immediate publication on acceptance

- Inclusion in PubMed, CAS, Scopus and Google Scholar

- Research which is freely available for redistribution 